\title{
A Notable Quasi-Relativistic Wave Equation and Its Relation to the Schrödinger, Klein-Gordon, and Dirac Equations
}

\author{
Luis Grave de Peralta ${ }^{1,2}$, Hira Farooq ${ }^{1}$ \\ ${ }^{1}$ Department of Physics and Astronomy, Texas Tech University, Lubbock, USA \\ ${ }^{2}$ Nano Tech Center, Texas Tech University, Lubbock, USA \\ Email: luis.grave-de-peralta@ttu.edu
}

How to cite this paper: de Peralta, L.G and Farooq, H. (2021) A Notable Quasi-Relativistic Wave Equation and Its Relation to the Schrödinger, Klein-Gordon, and Dirac Equations. Journal of Modern Physics, 12, 1145-1159.

https://doi.org/10.4236/jmp.2021.128068

Received: May 10, 2021

Accepted: June 5, 2021

Published: June 8, 2021

Copyright $\odot 2021$ by author(s) and Scientific Research Publishing Inc. This work is licensed under the Creative Commons Attribution International License (CC BY 4.0).

http://creativecommons.org/licenses/by/4.0/

(c) (i) Open Access

\begin{abstract}
An intriguing quasi-relativistic wave equation, which is useful between the range of applications of the Schrödinger and the Klein-Gordon equations, is discussed. This equation allows for a quantum description of a constant number of spin-0 particles moving at quasi-relativistic energies. It is shown how to obtain a Pauli-like version of this equation from the Dirac equation. This Pauli-like quasi-relativistic wave equation allows for a quantum description of a constant number of spin-1/2 particles moving at quasi-relativistic energies and interacting with an external electromagnetic field. In addition, it was found an excellent agreement between the energies of the electron in heavy Hydrogen-like atoms obtained using the Dirac equation, and the energies calculated using a perturbation approach based on the quasi-relativistic wave equation. Finally, it is argued that the notable quasi-relativistic wave equation discussed in this work provides interesting pedagogical opportunities for a fresh approach to the introduction to relativistic effects in introductory quantum mechanics courses.
\end{abstract}

\section{Keywords}

Quantum Mechanics, Schrödinger Equation, Klein-Gordon Equation, Dirac Equation, Relativistic Quantum Mechanics

\section{Introduction}

Most physicists are familiar with the Schrödinger equation, which describes the movement of a spin-0 particle with mass $(m)$ moving at speeds much smaller than the speed of light (c) [1] [2] [3] [4] [5]. The one-dimensional Schrödinger equation corresponding to a free particle is given by the following expression [1] 
[2] [3] [4] [5]:

$$
i \hbar \frac{\partial}{\partial t} \psi_{S c h}(x, t)=-\frac{\hbar^{2}}{2 m} \frac{\partial^{2}}{\partial x^{2}} \psi_{S c h}(x, t) .
$$

In Equation (1), $\hbar$ is the Plank constant $(h)$ divided by $2 \pi$ and $\psi_{\text {Sch }}$ is the (scalar) wavefunction. Most Physics Ph.D. graduates know about the KleinGordon equation, which describes the movement of a spin-0 particle with mass moving at relativistic speeds [6] [7]. The one-dimensional Klein-Gordon equation corresponding to a free particle is given by the following expression [6] [7]:

$$
\frac{1}{c^{2}} \frac{\partial^{2}}{\partial t^{2}} \psi_{K G}(x, t)=\frac{\partial^{2}}{\partial x^{2}} \psi_{K G}(x, t)-\frac{m^{2} c^{2}}{\hbar^{2}} \psi_{K G}(x, t) .
$$

In Equation (2), $\psi_{K G}$ is also a scalar wavefunction. Equation (2) is not a Schrödinger-like equation because in contrast to the Schrödinger equation, Equation (2) includes a second order temporal derivative. Introductory Quantum Mechanics courses often cover the Schrödinger equation [1] [2] [3] [4] [5]. More advance Quantum Mechanics courses often cover the Klein Gordon equation [6] [7]. This is done for introducing the readers to the consequences for quantum mechanics of taking seriously the concepts and ideas of Einstein's Special Theory of Relativity [8] [9].

Historically, while looking in 1926 for the right quantum equation, Erwin Schrödinger first explored, but did not publish, the equation that we today call the Klein-Gordon equation, which was also published in 1926 by Oskar Klein and Walter Gordon. Schrödinger was well-aware of the special theory of relativity; thus, he was looking for a Lorentz invariant wave equation [6] [7] [8] [9] [10]. The Schrödinger equation is not Lorentz invariant but Galilean invariant [10] [11]; therefore, a relativistic quantum mechanics cannot be based on the Schrödinger equation.

A fully relativistic quantum theory requires to be founded on equations like the Klein-Gordon equation, which is valid for any two observers moving respect to each other at constant velocity [6] [7]. However, judging by its popularity among present physicists, Schrödinger took the correct decision. The solutions of the Klein-Gordon equation are plagued with several unwanted properties that made Equation (2) less easy to work with than using Equation (1) [6] [7]. Equation (1) describes a particle of mass $(m)$, linear momentum $(p)$, and kinetic energy $(K)$ related by the classical relation $K=p^{2} / 2 m$, which is not valid at relativistic speeds [6] [7] [11].

Fortunately for Schrödinger, he was able to reproduce the results previously obtained by Bohr for the energies of the bounded states of the electron in the Hydrogen atom [1] [2] [3] [4] [5]. This was possible because the electron in the Hydrogen atom has non-relativistic energies [1] [2] [3] [4] [5]. However, electrons are not spin- 0 particles but spin-1/2 particles.

Electrons moving at low velocities respect to $c$, can be approximately described by a two-component vector wavefunction (spinor) [2] [6] [7]. The spinor 
nature of the electron wavefunction produces experimentally detectable results when the electron interacts with an external electromagnetic field [4] [6] [7]. The Pauli equation, which was discovered by Wolfgang Ernst Pauli in 1927, is a Schrödinger-like equation; therefore, it is not a Lorentz-invariant. The Pauli equation describing the interaction of a free electron with a constant magnetic field, with magnitude $B_{e x t}$ pointing in the $z$ direction, can be written in the following way [4]:

$$
i \hbar \frac{\partial}{\partial t} \psi_{P}(\boldsymbol{r}, t)=-\frac{\hbar^{2}}{2 m} \nabla^{2} \psi_{P}(\boldsymbol{r}, t)-\mu_{B} B_{e x t} \sigma_{z} \psi_{P}(\boldsymbol{r}, t) .
$$

In Equation (3), $\nabla^{2}$ is the Laplace operator [1] [2] [3] [4] [5], $\mu_{B}=e \hbar /(2 m c)$ is the Bohr magneton [4], $e$ is the electron charge, and $\sigma_{z}$ is the $2 \times 2$ Pauli matrix [2]:

$$
\sigma_{z}=\left(\begin{array}{cc}
1 & 0 \\
0 & -1
\end{array}\right)
$$

$\psi_{P}$ is not a scalar wavefunction but the two-component spinor wavefunction:

$$
\psi_{P}(\boldsymbol{r}, t)=\left(\begin{array}{l}
\psi_{P_{+}}(\boldsymbol{r}, t) \\
\psi_{P_{-}}(\boldsymbol{r}, t)
\end{array}\right) .
$$

Consequently, Equation (3) is equivalent to a system of two independent Schrödinger equations for $\psi_{P_{+}}$and $\psi_{P_{-}}$that are only different in the sign of the last term in the right side of the equations. When $B_{\text {ext }}=0$, both equations are equal to the three-dimensional version of Equation (1) [1] [2] [3] [4] [5]. The exact description of electrons moving at relativistic velocities requires a fourcomponent (biespinor) wavefunction, and the solution of the Lorentz invariant Dirac equation [6] [7]. The Dirac equation of a free electron is given by the following equation [2] [6] [7] [12]:

$$
i \hbar \frac{\partial}{\partial t} \psi_{D}(\boldsymbol{r}, t)=C[\hat{\boldsymbol{\alpha}} \cdot \hat{\boldsymbol{p}}] \psi_{D}(\boldsymbol{r}, t)+m c^{2} \hat{\beta} \psi_{D}(\boldsymbol{r}, t) .
$$

In Equation (6), each of the three components of the vector operator $\alpha$ and the operator $\beta$ are $4 \times 4$ Dirac's matrices [2] [6] [7] [14]. Each of the tree components of the linear momentum operator $p$ is the differential operator [2] [6] [7] [12].

$$
\hat{p}_{j}=-i \hbar \frac{\partial}{\partial j}, j=x, y, z .
$$

Consequently, the Dirac equation is not a Schrödinger-like equation because only includes spatial derivatives of first order, while Equations (1) and (3) include spatial derivatives of second order. The bispinor $\psi_{D}$ has four components; therefore, it can be represented using two spinors in the following way [2] [12]:

$$
\psi_{D}(\boldsymbol{r}, t)=\left(\begin{array}{c}
\varphi(\boldsymbol{r}, t) \\
\chi(\boldsymbol{r}, t)
\end{array}\right)
$$


Clearly, a price in mathematical complexity is paid for improving the relativistic description of quantum particles. Consequently, from a purely pedagogical point of view, it would be convenient to be able to have a Schrödinger-like equation capable to describe quantum-particles at relativistic energies. Unfortunately, this is not in general possible [6] [7]. Nevertheless, it was recently found a Schrödinger-like equation capable to describe quantum-particles at quasi-relativistic energies [11] [13] [14] [15] [16].

Rigorously, the number of particles may not be constant in a fully relativistic quantum theory [6] [7]. This is because when the sum of the kinetic and the potential $(U)$ energy of a particle with mass $m$ doubles the energy associate to the mass of the particle, i.e., $E^{\prime}=K+U=2 m c^{2}$, then a pair particle-antiparticle could be created from $E^{\prime}$ [2] [6] [7]. Consequently, the number of particles is constant at quasi-relativistic energies, i.e., when $E^{\prime}=K+U<2 m c^{2}$. At quasi-relativistic energies close to $m c^{2}$, the Schrödinger equation does not provide a good description of the states of the quantum particle because it assumes that $K=p^{2} / 2 m$, while at relativistic speeds the correct relation between $K, p$, and the square of the velocity of the particle $\left(v^{2}\right)$ is given by the following equation [8] [9] [11] [13] [14] [15] [16]:

$$
K=\frac{p^{2}}{\left(\gamma_{v}+1\right) m}, \gamma_{v}=\frac{1}{\sqrt{1-\frac{v^{2}}{c^{2}}}} .
$$

A free spin-0 particle can be (approximately) described by the following quasi-relativistic wave equation, which was first proposed by one of the authors of this work [11] [13] [14] [15] [16]:

$$
i \hbar \frac{\partial}{\partial t} \psi(x, t)=-\frac{\hbar^{2}}{\left(\gamma_{v}+1\right) m} \frac{\partial^{2}}{\partial x^{2}} \psi(x, t) .
$$

Clearly, Equation (10) is a Schrödinger-like equation. Like in Equation (1), $\psi$ is a scalar wavefunction. Moreover, Equation (10) coincides with Equation (1) at low velocities when $\gamma_{v} \sim 1$. However, Equation (10) describes a particle at quasi-relativistic energies because it implies the relation between $K, p$, and $v^{2}$ given by Equation (9) [11] [13] [14] [15] [16]. Consequently, from a purely pedagogical point of view, the quasi-relativistic wave equation (Equation (10)) is very interesting.

Moreover, the quasi-relativistic wave equation can be solved following the same mathematical steps required for solving the Schrödinger equation in most of the problems often included in Introductory Quantum Mechanics courses. This includes a free particle [11], confinement of a quantum particle in box [11] [14] [15], reflection by a sharp quantum potential [15], tunnel effect [15], and the quasi-relativistic description of Hydrogen-like atoms [14] [15] [16]. Therefore Equation (10) allows for a smooth introduction of special relativity concepts and ideas in Introductory Quantum Mechanics courses.

The quasi-relativistic wave equation also enriches the accumulated physics 
knowledge, and open new ways to tackle quantum problems involving particles at quasi-relativistic energies. Because Equation (10) is a Schrödinger-like equation, it permits to calculate probabilities like it is done for Equation (1) [11]. Moreover, Equation (10) allows for a quasi-relativistic description of multi-particle systems where the number of particles is constant [17]. This includes all problems in Chemistry where the number of electrons is constant and $E^{\prime}<2 m c^{2}$. The energy of the most energetic electrons in heavy elements is quasi-relativistic. Therefore, often their description either involves a perturbative theory based on the Schrödinger equation [2] [4] [5], or a more precise but much more complicate quantum electrodynamic description [18].

The quasi-relativistic wave equation potentially represents a novel non-perturbative approach for tackling such problems without having to pay a heavy price in mathematical complexity, thus helping to grasp the essence of the consequences of introducing the ideas and concepts of spatial theory of relativity in quantum mechanics.

In this work, first, for completeness, the connection between Equation (10) and the Klein-Gordon equation will be summarized. Then, for the first time, a quasi-relativistic version of Equation (3) will be directly obtained from the Dirac equation. Finally, also for the first time, an equation giving the quasi-relativistic energies of the bound states of the electron in Hydrogen-like atoms will be obtained using a perturbative approach based on the quasi-relativistic wave equation. The quasi-relativistic energies calculated in this way have a much better correspondence, with the energies calculated using the Dirac equation, than the energies calculated using a perturbative theory based on the Schrödinger equation.

\section{Relationship between the Klein-Gordon and the Quasi-Relativistic Wave Equations}

From the following well-known relativistic equations [8] [9] [15]:

$$
E^{2}-m^{2} c^{4}=p^{2} c^{2} \Leftrightarrow\left(E+m c^{2}\right)\left(E-m c^{2}\right)=p^{2} c^{2} .
$$

And:

$$
E=\gamma_{v} m c^{2}, p=\gamma_{v} m V, E=K+m c^{2} .
$$

One can formally obtain Equation (2) by substituting $E$ and $p$ in Equation (11) by the following energy and momentum quantum operators [1] [2] [3] [4] [6] [7]:

$$
\hat{E}=i \hbar \frac{\partial}{\partial t}, \hat{p}=-i \hbar \frac{\partial}{\partial x} .
$$

The factor $\left(E+m c^{2}\right)$ in Equation (11) is always different than zero for $E>0$; consequently, Equation (11) and the following algebraic equation are equivalents for $E>0$ :

$$
K=\left(E-m c^{2}\right)=\frac{p^{2}}{\left(\gamma_{v}+1\right) m} .
$$


Then from Equations (13) and (14) follow the following differential equation [13] [14] [15]:

$$
\hbar \frac{\partial}{\partial t} \psi_{K G+}(x, t)=-\frac{\hbar^{2}}{\left(\gamma_{v}+1\right) m} \frac{\partial^{2}}{\partial x^{2}} \psi_{K G+}(x, t)+m c^{2} \psi_{K G+}(x, t) .
$$

A simple substitution in Equations (2) and (15) shows that the following plane wave is a solution of both equations for $E>0$ :

$$
\psi_{K G+}(x, t)=\mathrm{e}^{\frac{i}{\hbar}(p x-E t)} .
$$

Moreover, the following wavefunction is a solution of Equation (10):

$$
\psi(x, t)=\psi_{K G+} \mathrm{e}^{i w_{m} t}, w_{m}=\frac{m c^{2}}{\hbar} .
$$

Therefore, Equation (17) allows finding a solution of Equation (2) with $E>0$ from a solution of Equation (10). This is the relationship between the freeparticle Klein-Gordon and quasi-relativistic wave equations. This relationship is also valid when the particle is moving through a potential $U$ [11] [13] [14] [15] [19]. For instance, the quasi-relativistic wave equation for a particle moving at quasi-relativistic energies through piecewise constant potentials is given by the following equation [15]:

$$
i \hbar \frac{\partial}{\partial t} \psi(x, t)=-\frac{\hbar^{2}}{\left(\gamma_{v}+1\right) m} \frac{\partial^{2}}{\partial x^{2}} \psi(x, t)+U(x) \psi(x, t) .
$$

Looking for a solution of Equation (18) of the form:

$$
\psi(x, t)=X(x) \mathrm{e}^{-\frac{i}{\hbar} K t}, K=E^{\prime}-U .
$$

It is obtained the time-independent quasi-relativistic wave equation [15]:

$$
\begin{aligned}
& \frac{\mathrm{d}^{2}}{\mathrm{~d} x^{2}} X(x)+\kappa^{2} X(x)=0 \\
& \kappa=\frac{p}{\hbar}=\frac{1}{\hbar} \sqrt{\left(\gamma_{v}+1\right) m K}=\frac{1}{\hbar} \sqrt{\left(\gamma_{v}+1\right) m\left(E^{\prime}-U\right)} .
\end{aligned}
$$

At low velocities, when $\gamma_{v} \sim 1$, Equation (1) coincides with the time-independent Schrödinger equation for the same problem [1] [2] [3] [4]. The allowed values of $\kappa$ are determined by the boundary conditions of the problem. From Equations (12) and (14) follows that [15] [17]:

$$
\gamma_{v}^{2}=1+\left(\frac{\hbar k}{m c}\right)^{2} \Rightarrow K=\frac{\hbar^{2} \kappa^{2}}{\left[1+\sqrt{1+\frac{\hbar}{m c} k}\right] m}
$$

At low velocities, when $\gamma_{v} \sim 1$ and $\hbar \kappa \ll m c$, Equation (21) gives $K=\hbar^{2} \kappa^{2} / 2 m$, which is the non-relativistic relation between $K$ and $\kappa$ [1] [2] [3] [4]. It is worth noting that Equations (10) and (18) are not linear equations [11] [13] [14] [15]. This may rise some objections due to the importance of the superposition principle in quantum mechanics [1]-[7] [11] [13] [14] [15]. How- 
ever, it should be noted that, if $X_{1}$ and $X_{2}$ are two solutions of the time-independent quasi-relativistic wave equation (Equation (20)), respectively corresponding to different kinetic energies $K_{1}$ and $K_{2}$, then the following wavefunction is a solution of the Klein-Gordon equation:

$$
\psi_{K G+}(x, t)=X_{1}(x) \mathrm{e}^{-\frac{i}{\hbar}\left(K_{1}+m c^{2}\right) t}+X_{2}(x) \mathrm{e}^{-\frac{i}{\hbar}\left(K_{2}+m c^{2}\right) t} .
$$

From this point of view, the time-independent relativistic wave equation should not be considered a fundamental equation, but a useful auxiliar equation for finding solutions of a fundamental Lorentz invariant wave equation satisfying the superposition principle [14].

\section{The Quasi-Relativistic Wave Equation of a Free Electron}

The wavefunction in Equations (1), (2), (10), and (18) are scalars, thus describe the state of a spin-0 particle with mass. However, electrons are not spin-0 particles but spin-1/2 particles. Equation (6) gives the correct relativistic equation of a free electron. However, as it is shown below, a spinor quasi-relativistic wave equation can be obtained when $E>0$. Proposing a solution of Equation (6) of the following form [2]:

$$
\psi_{D}(\boldsymbol{r}, t)=\left(\begin{array}{l}
\varphi(\boldsymbol{r}) \\
\chi(\boldsymbol{r})
\end{array}\right) \mathrm{e}^{-\frac{i}{\hbar} E t} .
$$

Substituting Equation (23) in Equation (6), and considering that for a free electron $E=K+m c^{2}$, allows for rewriting Equation (6) as the following system of two time-independent spinor equations [2]:

$$
c[\hat{\boldsymbol{\sigma}} \cdot \hat{\boldsymbol{p}}] \chi=\left(E-m c^{2}\right) \varphi=K \varphi, c[\hat{\boldsymbol{\sigma}} \cdot \hat{\boldsymbol{p}}] \varphi=\left(E+m c^{2}\right) \chi .
$$

In Equation (24), each of the three components of the vector operator $\sigma$ is a $2 \times 2$ Pauli's matrix [2] [6] [7] [14]. $E+m c^{2}>0$ when $E>0$, thus when $E>0$, the second equation of Equation (24) can be rewritten in the following way:

$$
\chi=\frac{c[\hat{\boldsymbol{\sigma}} \cdot \hat{\boldsymbol{p}}]}{\left(E+m c^{2}\right)} \varphi=\frac{[\hat{\boldsymbol{\sigma}} \cdot \hat{\boldsymbol{p}}]}{\left(\gamma_{v}+1\right) m c} \varphi
$$

Substituting Equation (25) in the first equation of Equation (24) results in the following equation:

$$
\frac{[\hat{\boldsymbol{\sigma}} \cdot \hat{\boldsymbol{p}}]^{2}}{\left(\gamma_{v}+1\right) m} \varphi=-\frac{\hbar^{2}}{\left(\gamma_{v}+1\right) m} \nabla^{2} \varphi=K \varphi .
$$

Therefore, when $E>0$, each one of the two components of $\varphi$ exactly satisfies the same time-independent quasi-relativistic wave equation, which corresponds to a free spin-0 particle with kinetic energy $K$. Consequently, when $E>0$, the three-dimensional version of Equation (10) is the time-dependent quasi-relativist wave equation corresponding to each component of $\varphi$ in Equation (26). 


\section{The Pauli-Like Quasi-Relativistic Wave Equation}

The Schrödinger-like Pauli equation given by Equation (3) can be obtained from the Dirac equation for an electron interacting with an external electromagnetic field [2]. Following the same procedure, a quasi-relativistic version of Equation (3) can be obtained. When an external electromagnetic field interact with the electron, Equation (24) should be modified in the following way [2]:

$$
c\left[\hat{\boldsymbol{\sigma}} \cdot \hat{\boldsymbol{p}}-\frac{e}{c} \boldsymbol{A}\right] \chi=\left(E-m c^{2}-e A_{o}\right) \varphi, c\left[\hat{\boldsymbol{\sigma}} \cdot \hat{\boldsymbol{p}}-\frac{e}{c} \boldsymbol{A}\right] \varphi=\left(E+m c^{2}-e A_{o}\right) \chi \cdot(
$$

In Equation (27), $-e A_{o}$ is the electron electrostatic energy and the vector potential $A$ is associated to an external magnetic field [2] [8]. When

$\left(E+m c^{2}-e A_{o}\right)>0$, the second equation of Equation (27) can be rewritten in the following way:

$$
\chi=\frac{c\left[\hat{\boldsymbol{\sigma}} \cdot \hat{\boldsymbol{p}}-\frac{e}{c} \boldsymbol{A}\right]}{\left(E+m c^{2}-e A_{o}\right)} \varphi .
$$

The Schrödinger-like Pauli equation can be obtained doing $E=E^{\prime}+m c^{2}$ and assuming $\left|E^{\prime}-e A_{o}\right| \ll m c^{2}$. Therefore, the fraction $c /\left(E^{\prime}-e A_{o}+2 m c^{2}\right)$ in Equation (28) can be developed in powers of $\left(E^{\prime}-e A_{o}\right)$ and Equation (28) can be approximated by the following expression [2]:

$$
\chi \approx \frac{1}{2 m}\left[\hat{\boldsymbol{\sigma}} \cdot \hat{\boldsymbol{p}}-\frac{e}{c} \boldsymbol{A}\right] \varphi
$$

Substituting Equation (29) in the first equation of Equation (27) allows obtaining the Schrödinger-like time-independent Pauli equation [2]:

$$
\left\{\frac{\left[\hat{\boldsymbol{p}}-\frac{e}{c} \boldsymbol{A}\right]^{2}}{2 m}+e A_{o}-\mu_{B}(\hat{\boldsymbol{\sigma}} \cdot \vec{B})\right\} \varphi=E^{\prime} \varphi .
$$

For a free electron moving through a constant magnetic field, with magnitude $B_{\text {ext }}$ pointing in the $z$ direction, Equation (30) can be approximated as:

$$
-\frac{\hbar^{2}}{2 m} \nabla^{2} \varphi(\boldsymbol{r})-\mu_{B} B_{e x t} \sigma_{z} \varphi(\boldsymbol{r})=E^{\prime} \varphi
$$

Which is the time-independent Pauli-equation corresponding to Equation (3). However, if one assumed that $\left|-e A_{o}\right| \ll E+m c^{2}$, then the fraction $c /\left(-e A_{o}+E^{\prime}+2 m c^{2}\right)$ in Equation (28) can be developed in powers of $-e A_{o}$ and Equation (28) can be approximated by the following expression:

$$
\chi \approx \frac{1}{\left(\gamma_{v}+1\right) m c}\left[\hat{\boldsymbol{\sigma}} \cdot \hat{\boldsymbol{p}}-\frac{e}{c} \boldsymbol{A}\right] \varphi .
$$

Substituting Equation (32) in the first equation of Equation (27) allows obtaining the following time-independent Pauli-like quasi-relativistic wave equation: 


$$
\left\{\frac{\left[\hat{\boldsymbol{p}}-\frac{e}{c} \boldsymbol{A}\right]^{2}}{\left(\gamma_{v}+1\right) m}+e A_{o}-\frac{2 \mu_{B}}{\left(\gamma_{V}+1\right)}(\hat{\boldsymbol{\sigma}} \cdot \boldsymbol{B})\right\} \varphi=E^{\prime} \varphi .
$$

For a free electron moving through a constant magnetic field, with magnitude $B_{e x t}$ pointing in the $z$ direction, Equation (33) can be approximated as:

$$
-\frac{\hbar^{2}}{\left(\gamma_{v}+1\right) m} \nabla^{2} \varphi(\boldsymbol{r})-\frac{2 \mu_{B}}{\left(\gamma_{v}+1\right)} B_{e x t} \sigma_{z} \varphi(\boldsymbol{r})=E^{\prime} \varphi .
$$

Equation (34) is the quasi-relativistic version of Equation (31). When the electron moves slowly, $\gamma_{v} \sim 1$, thus Equation (34) coincides with Equation (31). Equation (34) includes two corrections to Equation (3). First, includes the correct relativistic relation between $K$ and $p$. Second, as shown in Figure 1, the energy difference corresponding to the two components of $\varphi$ is not independent of $K$, as suggested by Equation (31), but decreases by a factor of twice $2 /\left(\gamma_{v}+1\right)$ at quasi-relativistic energies. This relevant result could be easily tested experimentally.

\section{Relativistic Corrections to the Energies of the Bounded States in Hydrogen-Like Atoms}

For Hydrogen-like atoms, we can assume the vector potential in Equation (27) is null, and:

$$
e A_{o}=U_{C}(r)=-\frac{e^{2}}{4 \pi \varepsilon_{o}} \frac{Z}{r} .
$$

In Equation (35), $U_{C}$ is the Coulombic electrostatic energy, $Z$ is the atomic number, and $\varepsilon_{o}$ is the electric permittivity of vacuum [2] [4] [13] [15]. The exact Dirac's energies of the bound states of the electron in Hydrogen-like atoms are given by the following equation [2]:

$$
\begin{aligned}
& E^{\prime}=\mu c^{2}\left[1+\left(\frac{Z \alpha}{n-\left(j+\frac{1}{2}\right)+\sqrt{\left(j+\frac{1}{2}\right)-Z^{2} \alpha^{2}}}\right]^{2}\right]^{-\frac{1}{2}}-\mu c^{2} .
\end{aligned}
$$

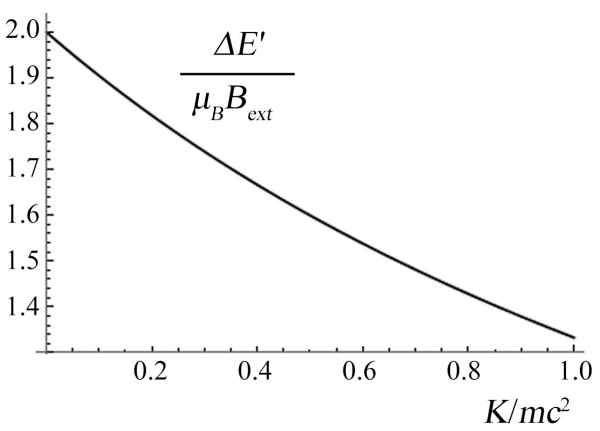

Figure 1. Plot oftwice $2 /\left(\gamma_{\mathrm{v}}+1\right)$ as a function of $K$ in $m c^{2}$ units. 
In Equation (36), $n=1,2, \cdots ; l=0,1, \cdots,(n-1) ; j=l \pm 1 / 2$, $\alpha=\left(1 / 4 \pi \varepsilon_{0}\right) \times\left(e^{2} / \hbar c\right) \approx 1 / 137$ is the fine structure constant, $\mu=\left(m_{e} m_{n}\right) /\left(m_{e}+m_{n}\right)$ is the reduced mass of the electron in a Hydrogen-like atom with a nucleus of mass $m_{n}$, and $m_{e}$ is the electron mass [2]. Often the following approximation to Equation (36), which is valid when $E^{\prime} \ll m c^{2}$, is obtained using a perturbative approach based in the Schrödinger equation [2] [12]:

$$
E^{\prime}=E_{S c h}\left(1+\Delta E_{K, S c h}+\Delta E_{D, S c h}+\Delta E_{S O, S c h}\right) .
$$

In Equation (37), $E_{S c h}$ gives the values, of the bounded energies of the electron in Hydrogen-like atoms, obtained using the Schrödinger equation [1] [2] [3] [4] [5] [14]:

$$
E_{S c h}=-\left[\frac{\mu}{2 \hbar^{2}}\left(\frac{e^{2}}{4 \pi \varepsilon_{o}}\right)^{2}\right] \frac{Z^{2}}{n^{2}}=-\frac{\mu c^{2}}{4} \frac{\alpha^{2} Z^{2}}{n^{2}} .
$$

$\Delta E_{K, S c h}$ is the relativistic correction to the kinetic energy, which is given by the following expression [2] [11] [12] [13] [14]:

$$
\Delta E_{K, S c h}=-E_{S c h} \frac{\alpha^{2} Z^{2}}{n^{2}}\left(\frac{3}{4}-\frac{n}{l+\frac{1}{2}}\right) .
$$

$\Delta E_{D, S c h}$ is the so-called the Darwin correction, which is only not null when $l=0 \quad[2][12]:$

$$
\Delta E_{D, S c h}=-E_{S c h} \frac{\alpha^{2} Z^{2}}{n^{2}} .
$$

Finally, $\Delta E_{S O, S c h}$ is the so-called spin-orbit correction, which is only not null when $l \neq 0 \quad[2][12]$ :

$$
\Delta E_{D, S c h}=-E_{S c h} \frac{\alpha^{2} Z^{2}}{2 n} \frac{j(j+1)-l(l+1)+3 / 4}{l\left(l+\frac{1}{2}\right)(l+1)} .
$$

From Equations (38) to (41) follows the relativistic corrections are much smaller that $E_{S c h}$ when $(\alpha Z / n)^{2} \ll 1$. One should expect the energies calculated using Equation (37) sensibly differ from the exact Dirac's energies for the lowest energy states (smallest $n$-values) of heavy Hydrogen-like atoms. At this point, however, no one should be surprised by the fact that following a similar procedure than the used for obtaining Equation (37), but using a perturbative approach based in the quasi-relativistic wave equation (details shown in the Appendix), one can find a much better approximation to Equation (36), which is valid until quasi-relativistic energies:

$$
E^{\prime}=E_{Q R}\left(1+\Delta E_{D, Q R}+\Delta E_{S O, Q R}\right) .
$$

In Equation (42), $E_{Q R}$ gives the energies of the bounded states obtained using the quasi-relativistic wave equation for Hydrogen-like atoms [15]: 


$$
E_{Q R}=-\frac{\mu c^{2}}{\Xi}[\Xi-(2 n+\Delta) \sqrt{\Xi}] .
$$

In Equation (43), $\Delta=\Delta(l, Z)$ and $\Xi$ are given by the following equations [15]:

$$
\Xi=4 n^{2}+4 \alpha^{2} Z^{2}+4 n \Delta+\Delta^{2} .
$$

And:

$$
\begin{aligned}
& \Delta(l, Z)=\left[\left(1+\sqrt{(1+2 l)^{2}-4 \alpha^{2} Z^{2}}\right)-2(l+1)\right], \\
& \sqrt{(1+2 l)^{2}-4 \alpha^{2} Z^{2}} \approx(1+2 l)-\frac{2 \alpha^{2} Z^{2}}{(1+2 l)}-\frac{2 \alpha^{4} Z^{4}}{(1+2 l)^{3}} .
\end{aligned}
$$

In some cases, for heavy Hydrogen-like atoms with $Z \gg 1$, the term inside the square root in Equation (45) could be negative; in these cases, the approximation to the square root included in Equation (45) should be used. As should be expected, $E_{Q R} \approx E_{S c h}\left(1+\Delta E_{K, S c h}\right)$ when $E^{\prime} \ll m c^{2}$ [15]. It is worth noting that $E_{Q R}$ is identical to the positive energies calculated for the Hydrogen atom using the Klein-Gordon equation [19]. $\Delta E_{D, Q R}$ is the new Darwin correction, which also is only not null when $l=0$ :

$$
\Delta E_{D, Q R}=-k_{D} E_{Q R} \frac{\alpha^{2} Z^{2}}{n}, k_{D}=\left(\gamma_{v}+1\right)^{\frac{n}{n+1}} .
$$

$\Delta E_{S O, Q D}$ is the new spin-orbit correction, which also is only not null when $l \neq 0$ :

$$
\Delta E_{D, S c h}=-k_{S O} E_{Q R} \frac{\alpha^{2} Z^{2}}{2 n} \frac{j(j+1)-l(l+1)+\frac{3}{4}}{l\left(l+\frac{1}{2}\right)(l+1)}, k_{S O}=\left(\frac{\gamma_{V}+1}{2}\right)^{-(n-l+1)^{5 / 2}} .
$$

The energies of the ground state $(n=1, l=0, j=1 / 2)$ of the Hydrogen atom $(Z=1)$ calculated using Equations (36), (37), (38), (42), and (43) are $E^{\prime}=$ $-13.6022,-13.6022,-13.6020,-13.6019$, and $-13.6029 \mathrm{eV}$, respectively. All these values are within a $0.005 \%$ error respect to the exact Dirac's energy. This is because $E^{\prime} \ll m c^{2}$ when $Z=1$. A comparison between the calculated values of the energy difference between two emission lines $\left(\Delta E_{L}\right)$ of the Hydrogen atom are shown in Table 1. $\Delta E_{L}$ was calculated using the following equation:

$$
\begin{aligned}
& \Delta E_{L}=\left[E^{\prime}\left(n_{2}, l_{2}, j_{2}=l_{2}+\frac{1}{2}\right)-E^{\prime}\left(n_{1}, l_{1}, j_{1}=l_{1}+\frac{1}{2}\right)\right] \\
& -\left[E^{\prime}\left(n_{2}, l_{2}, j_{2}=l_{2}-\frac{1}{2}\right)-E^{\prime}\left(n_{1}, l_{1}, j_{1}=l_{1}+\frac{1}{2}\right)\right] .
\end{aligned}
$$

$E^{\prime}$ was evaluated using Equations (36), (37), and (42). For the $\alpha$-Lyman doublet, we used $n_{2}=2, l_{2}=1$ and $n_{1}=1, l_{1}=0$ [2] [12]. For the $\alpha$-Balmer doublet, we used $n_{2}=3, l_{2}=1$ and $n_{1}=2, l_{1}=0$ [2] [12]. The last column of Table 1 corresponds to $n_{2}=3, l_{2}=2$ and $n_{1}=2, l_{1}=1$. It was chosen as an instance where both $l_{2}$ and $l_{1}$ are not zero. In all instances in Table 1 , there is an excellent correspondence between the calculated values. Again, this is because $E^{\prime} \ll m c^{2}$ when $Z=1$. 
Table 1. Calculated values of $\Delta E_{L}$ (in $\mathrm{meV}$ ) for the Hydrogen atom obtained using Equations (36), (37), and (42) for (a) $\alpha$-Lyman doublet, (b) $\alpha$-Balmer doublet, and (c) corresponding to the energy difference between two others emission lines.

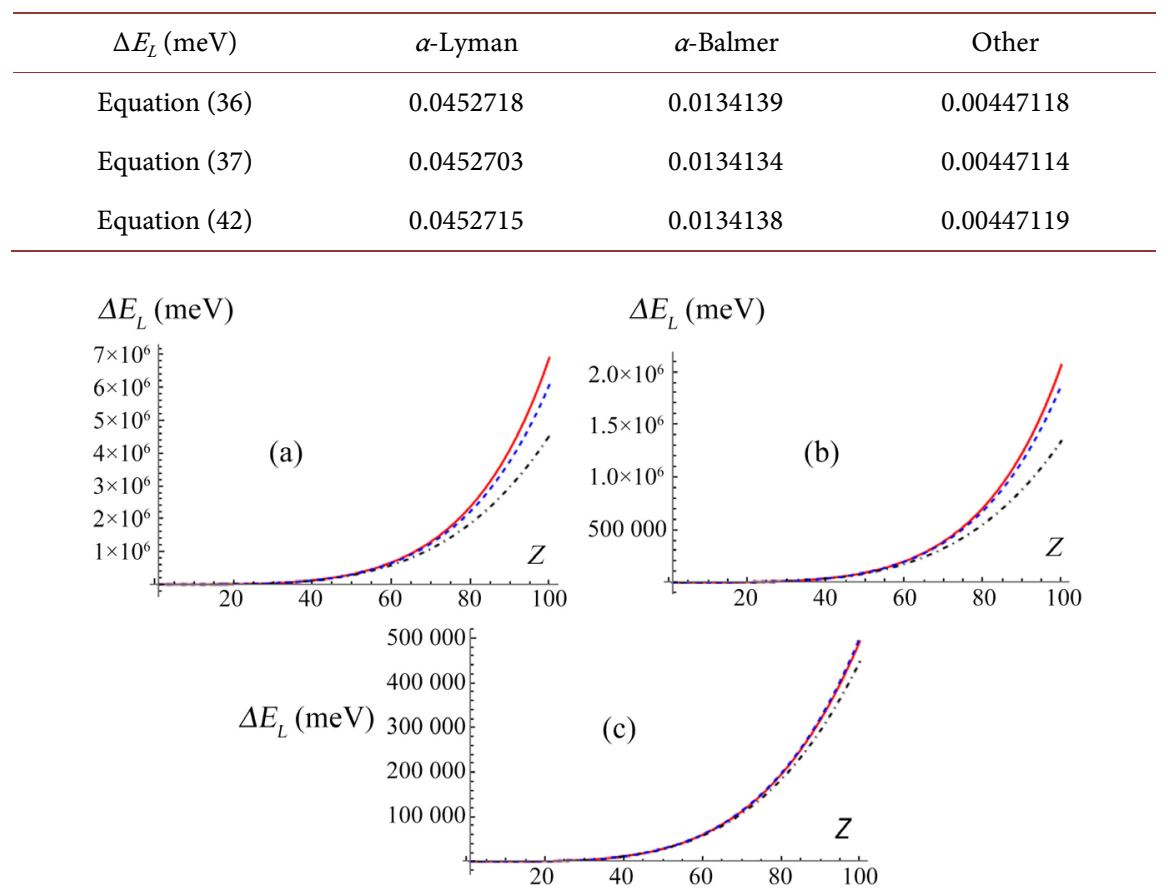

Figure 2. Dependence on $Z$ of $\Delta E_{L}$ (in meV) calculated using (red, continuous) Equation (36), (black, dot-dashed,) Equation (37), and (blue, dashed) Equation (42) for (a) $\alpha$-Lyman doublet, (b) $\alpha$-Balmer doublet, (c) another example corresponding to the last column of Table 1.

More importantly, Equation (42) provides a better approximation than Equation (37) to the values of $\Delta E_{L}$ calculated using Equation (36). This is confirmed by the plots shown in Figure 2 showing the dependence on $Z$ of $\Delta E_{L}$. Clearly, as expected, at quasi-relativistic energies ( $Z \gg 1$ ), Equation (42) (dashed blue curve) provides a much better approximation than Equation (37) (dot-dashed black curve) to the values of $\Delta E_{L}$ calculated using the exact Dirac's energies (continuous red curve).

\section{Conclusion}

It was shown that the time dependent Equations (1) and (18), and the time-independent Equation (20) are very useful equations which are directly related to the Klein-Gordon equation, thus allowing a quantum description of a constant number of spin- 0 particles moving at quasi-relativistic energies. It was presented and discussed, for the first time, a Pauli-like quasi-relativistic wave equation which is directly related to the Dirac equation, thus allowing for a quantum description of a constant number of spin-1/2 particles moving at quasi-relativistic energies and interacting with an external electromagnetic field. Finally, using a perturbative approach based on the quasi-relativistic wave equations discussed in this work, it was found and validated, also for the first time, an equation giv- 
ing the energies of the bounded states in Hydrogen-like atoms. The authors hope we have been able to motivate the curiosity of the readers. Undoubtedly, the equations and methods discussed here enrich the accumulated physics knowledge, and open new ways to tackle quantum problems involving a constant number of particles at quasi-relativistic energies. This also provides interesting pedagogical opportunities for a fresh approach to the introduction of relativistic effect in introductory quantum mechanics courses.

\section{Conflicts of Interest}

The authors declare no conflicts of interest regarding the publication of this paper.

\section{References}

[1] Bohm, D. (1964) Quantum Theory. 11th Edition, Prentice-Hall, New York.

[2] Davydov, A.S. (1965) Quantum Mechanics. Pergamon Press, New York.

[3] Merzbacher, E. (1970) Quantum Mechanics. 2nd Edition, J. Wiley \& Sons, New York.

[4] Griffiths, D.J. (1995) Introduction to Quantum Mechanics. Prentice Hall, New York.

[5] Levine, I.N. (2014) Quantum Chemistry. 7th Edition, Pearson Education, New York.

[6] Strange, P. (1998) Relativistic Quantum Mechanics: With Applications in Condensed Matter and Atomic Physics. Cambridge University Press, New York. https://doi.org/10.1017/CBO9780511622755

[7] Greiner, W. (1990) Relativistic Quantum Mechanics: Wave Equations. Spring-Verlag, New York

[8] Jackson, J.D. (1975) Classical Electrodynamics. 2nd Edition, J. Wiley \& Sons, New York.

[9] Steane, A.M. (2012) Relativity Made Relatively Easy. Oxford University Press, Oxford.

[10] Home, D. (1997) Conceptual Foundations of Quantum Physics: An Overview from Modern Perspectives. Plenum Press, New York.

[11] Grave de Peralta, L. (2020) Journal of Modern Physics, 11, 196-213. https://doi.org/10.4236/jmp.2020.112012

[12] Nanni, L. (2015) The Hydrogen Atom: A Review on the Birth of Modern Quantum Mechanics.

[13] Grave de Peralta, L. (2020) Journal of Modern Physics, 11, 788-802. https://doi.org/10.4236/jmp.2020.116051

[14] Grave de Peralta, L. (2020) European Journal of Physics, 41, Article ID: 065404. https://doi.org/10.1088/1361-6404/aba7dc

[15] Grave de Peralta, L. (2020) Results in Physics, 18, Article ID: 103318. https://doi.org/10.1016/j.rinp.2020.103318

[16] Grave de Peralta, L. (2020) Scientific Reports, 10, Article No. 14925. https://doi.org/10.1038/s41598-020-71505-w

[17] Poirier, B. (2020) Personal Communication. Department of Chemistry and Bio- 
chemistry, Texas Tech University, Lubbock.

[18] Griner, W. and Reinhardt, J. (2009) Quantum Electrodynamics. 4th Edition, Springer-Verlag, Berlin.

[19] Poveda, L.A. (2021) Personal Communication. Departemento de Fisica, Centro Federal de Educação Tecnológica de Minas Gerais, Amazonas 5253, 30421-169 Belo Horizonte, MG, Brasil. 


\section{Appendix}

Equations (37) and (42) can both be obtained from Equation (27) with a null vectorial potential $(A)$ and $e A_{o}$ given by Equation (35). For obtaining Equation (37), Equation (28) should be approximated in the following way [2]:

$$
\chi \approx \frac{1}{2 m c}\left(1-\frac{E^{\prime}-U_{C}}{2 m c^{2}}\right)[\hat{\boldsymbol{\sigma}} \cdot \hat{\boldsymbol{p}}] \varphi .
$$

Then, substituting Equation (A1) in the first equation of Equation (27) results [2]:

$$
\frac{1}{2 m}[\hat{\boldsymbol{\sigma}} \cdot \hat{\boldsymbol{p}}]\left(1-\frac{E^{\prime}-U_{C}}{2 m c^{2}}\right)[\hat{\boldsymbol{\sigma}} \cdot \hat{\boldsymbol{p}}] \varphi=\left[E^{\prime}-U_{C}(r)\right] \varphi .
$$

Or:

$$
\left[-\frac{\hbar^{2}}{2 m} \nabla^{2} \varphi+U_{C}(r)\right] \varphi-\left\{\frac{1}{2 m}[\hat{\boldsymbol{\sigma}} \cdot \hat{\boldsymbol{p}}]\left[\frac{E^{\prime}-U_{C}(r)}{2 m c^{2}}\right][\hat{\boldsymbol{\sigma}} \cdot \hat{\boldsymbol{p}}]\right\} \varphi=E^{\prime} \varphi .
$$

The time-independent Schrödinger equation for Hydrogen-like atoms is equal to Equation (A3) after excluding the term between curls in the left size of Equation (A3) [1] [2] [3] [4] [5]; therefore, the relativistic corrections to the energies calculated using the Schrödinger equation are contained in this term [2]. However, if Equation (28) is approximated in the following way:

$$
\chi \approx \frac{1}{\left(\gamma_{v}+1\right) m c}\left(1+\frac{U_{C}}{\left(\gamma_{v}+1\right) m c^{2}}\right)[\hat{\boldsymbol{\sigma}} \cdot \hat{\boldsymbol{p}}] \varphi .
$$

Then, substituting Equation (A4) in the first equation of Equation (27) results:

$$
\frac{1}{\left(\gamma_{v}+1\right) m}[\hat{\boldsymbol{\sigma}} \cdot \hat{\boldsymbol{p}}]\left(1+\frac{U_{C}}{\left(\gamma_{v}+1\right) m c^{2}}\right)[\hat{\boldsymbol{\sigma}} \cdot \hat{\boldsymbol{p}}] \varphi\left[E^{\prime}-U_{C}(r)\right] \varphi \text {. }
$$

Or:

$$
\left[-\frac{\hbar^{2}}{\left(\gamma_{v}+1\right) m} \nabla^{2} \varphi+U_{C}(r)\right] \varphi+\left\{\frac{1}{\left(\gamma_{v}+1\right) m}[\hat{\boldsymbol{\sigma}} \cdot \hat{\boldsymbol{p}}]\left[\frac{U_{C}(r)}{\left(\gamma_{v}+1\right) m c^{2}}\right][\hat{\boldsymbol{\sigma}} \cdot \hat{\boldsymbol{p}}]\right\} \varphi=E^{\prime} \varphi .
$$

The time-independent quasi-relativistic wave equation for Hydrogen-like atoms is equal to Equation (A6) after excluding the term between curls in the left size of Equation (A6) [12]; therefore, the relativistic corrections to the energies calculated using the quasi-relativistic wave equation are contained in this term. In Equation (A3), the term between curls produces three relativistic corrections to the energy, which are given by Equations (39) to (41) [2]. It can be shown, following the same procedure [2], but using the wavefunctions satisfying the quasi-relativistic wave equation for Hydrogen-like atoms [14] [16], that the term between curls in Equation (A6) produces two relativistic corrections to the energy, which are given by Equations (46) and (47). 